\title{
Article \\ Massive Spread of OXA-48 Carbapenemase-Producing Enterobacteriaceae in the Environment of a Swiss Companion Animal Clinic
}

\author{
Kira Schmitt ${ }^{1,2} \mathbb{D}$, Michael Biggel ${ }^{1}$, Roger Stephan ${ }^{1, *(\mathbb{D})}$ and Barbara Willi ${ }^{3} \mathbb{D}$ \\ 1 Institute for Food Safety and Hygiene, University of Zurich, CH-8057 Zurich, Switzerland; \\ kira.schmitt@uzh.ch (K.S.); michael.biggel@uzh.ch (M.B.) \\ 2 Graduate School for Cellular and Biomedical Sciences, University of Bern, CH-3012 Bern, Switzerland \\ 3 Clinic for Small Animal Internal Medicine, University of Zurich, $\mathrm{CH}-8057$ Zurich, Switzerland; \\ bwilli@vetclinics.uzh.ch \\ * Correspondence: roger.stephan@uzh.ch; Tel.: +41-44-635-86-51
}

check for

updates

Citation: Schmitt, K.; Biggel, M.; Stephan, R.; Willi, B. Massive Spread of OXA-48 Carbapenemase-Producing Enterobacteriaceae in the Environment of a Swiss Companion Animal Clinic. Antibiotics 2022, 11, 213. https:// doi.org/10.3390/antibiotics11020213 Academic Editors: Teresa Fasciana, Mara Di Giulio, Silvia Di Lodovico and Alberto Antonelli

Received: 30 December 2021

Accepted: 4 February 2022

Published: 8 February 2022

Publisher's Note: MDPI stays neutral with regard to jurisdictional claims in published maps and institutional affiliations.

Copyright: () 2022 by the authors. Licensee MDPI, Basel, Switzerland. This article is an open access article distributed under the terms and conditions of the Creative Commons Attribution (CC BY) license (https:/ / creativecommons.org/licenses/by/ $4.0 /)$.

\begin{abstract}
Background: Companion animal clinics contribute to the spread of antimicrobial resistant microorganisms (ARM) and outbreaks with ARM of public health concern have been described. Methods: As part of a project to assess infection prevention and control (IPC) standards in companion animal clinics in Switzerland, a total of 200 swabs from surfaces and 20 hand swabs from employees were collected during four days in a medium-sized clinic and analyzed for extended spectrum betalactamase-producing Enterobacteriaceae (ESBL-E), carbapenemase-producing Enterobacteriaceae (CPE), vancomycin-resistant enterococci (VRE), and methicillin-resistant staphylococci (MRS). Results: A total of 22 (11.0\%) environmental specimen yielded CPE, 14 (7.0\%) ESBL-E, and 7 (3.5\%) MRS; MR Staphylococcus aureus were isolated from two (10.0\%) hand swabs. The CPE isolates comprised Escherichia coli, Klebsiella pneumoniae, Enterobacter hormaechei, Citrobacter braakii, and Serratia marcescens. Whole genome sequencing revealed that all CPE carried closely related bla $a_{\mathrm{OXA}-48}$ plasmids, suggesting a plasmidic spread within the clinic. The clinic exhibited major deficits in surface disinfection, hand hygiene infrastructure, and hand hygiene compliance. CPE were present in various areas, including those without patient contact. The study documented plasmidic dissemination of $b l a_{\mathrm{OXA}-48}$ in a companion animal clinic with low IPC standards. This poses a worrisome threat to public health and highlights the need to foster IPC standards in veterinary clinics to prevent the spread of ARM into the community.
\end{abstract}

Keywords: CPE; CTX-M15; ESBL; IPC; hand hygiene; carbapenemase; OXA-48; IncL; plasmid

\section{Introduction}

Antimicrobial resistance has been declared as one of "the greatest and most urgent global risks" by the United Nations General Assembly [1]. Antimicrobial resistant microorganisms (ARM) are estimated to cause the death of over 700,000 people per year and pose a threat to the healthcare system [2]. Carbapenemase-producing Enterobacteriaceae (CPE) represent ARM of special concern due to their ability to hydrolyze carbapenems. The World Health Organization (WHO, Geneva, Switzerland) classifies carbapenem antibiotics as critically important for human health and considers them as antimicrobials of last resort due to their broad spectrum of activity against several gram-positive and gram-negative bacteria [3]. Carbapenem resistance encoded by bla $a_{\mathrm{OXA}-48}$ is often located on transferable L/M complex plasmids. These plasmids can possess derepressed transfer properties, allowing them to efficiently spread horizontally [4]. CPE have caused outbreaks and hospital-acquired infections in human healthcare worldwide [5-11]. In companion animal clinics, CPE have, so far, only scarcely been reported [12-15]. Of note, the use of carbapenems in companion animal medicine is restricted to special indications and has not 
been widely reported [16]. Companion animal clinics harbor multiple factors that foster the selection of antimicrobial resistant microorganisms (ARM): a high number of hospitalized and debilitated patients, a high percentage of animals receiving antimicrobial therapy, and daily invasive procedures associated with numerous hand-patient contacts.

As part of an action plan to combat the spread of ARM in companion animal clinics, a project to evaluate the effect of infection prevention and control (IPC) implementation in five veterinary clinics on environmental contamination with ARM, hand hygiene, and IPC standards was launched in Switzerland. During this project, a spread of CPE and extended-spectrum beta-lactamase producing Enterobacteriaceae (ESBL-E) was identified in one of the clinics which showed extensive environmental contamination. The aims of the present study were to characterize the isolates, to investigate potential transmission events, and to assess IPC standards in the affected institution.

\section{Results}

\subsection{IPC Standards and Hand Hygiene Adherence}

The dissemination of resistant bacteria was identified in a medium-sized clinic in Switzerland that comprised 45 staff members, offered a 24/7 emergency service and an intensive care unit (ICU), and treated around 13,000 ambulatory and stationary dogs and cats per year. In the IPC standard assessment the clinic reached 59 out of 102 (58\%) total scoring points (Table 1). The IPC audit revealed major deficits regarding cleaning and disinfection, hand hygiene infrastructure, isolation measures, equipment in examination rooms, and antimicrobial use. Written protocols on cleaning and disinfection were not implemented. Hand washing stations and hand disinfection dispensers were not available in all areas where patients were treated. The clinic had limited IPC management in place and staff education regarding IPC was scarce.

Table 1. IPC areas and audit scores.

\begin{tabular}{cc}
\hline IPC Area & IPC Audit Score/Maximum IPC Score \\
\hline IPC management & $1 / 10$ \\
Staff education & $5 / 12$ \\
Cleaning/disinfection & $5 / 8$ \\
Management of waste & $4 / 4$ \\
Vector control & $2 / 2$ \\
Equipment in examination rooms & $2 / 4$ \\
Isolation measures & $3 / 6$ \\
Patients with ARM & $3 / 4$ \\
Hand hygiene & $4 / 8$ \\
Personal hygiene & $10 / 12$ \\
Protection of employees & $5 / 8$ \\
Protective clothing & $5 / 6$ \\
Medication & $5 / 6$ \\
Use of antimicrobials & $2 / 4$ \\
Miscellaneous & $3 / 8$ \\
\hline Total audit score/maximum IPC score (\%) & $59 / 102(58 \%)$ \\
\hline Abbreviations: IPC, infection prevention and control; ARM, antimicrobial resistant microorganisms.
\end{tabular}

Overall, 525 hand hygiene observations were collected in the clinic. Overall hand hygiene compliance was 14.9\% (95\% CI 12.1-18.2\%). Significant differences were observed between clinical areas $(p=0.007)$ and hand hygiene indications $(p<0.0001)$. The highest hand hygiene compliance was found in the wards (23.9\%, 95\% CI 17.5-31.8\%), followed by the consultation area $(13.4 \%, 95 \%$ CI $8.5-20.4 \%)$, the ICU $(11.8 \%, 95 \%$ CI $7.3-18.6 \%)$, and the pre-operation preparation area (10.2\%, 95\% CI 6.2-16.4\%). Hand hygiene was more commonly performed after body fluid exposure risk (19.0\%, 95\% CI 12.9-27.0\%), after patient contact $(16.5 \%, 95 \%$ CI 11.2-23.8\%), and after touching the patient's surrounding 
(18.2\%, 95\% CI 11.8-26.9\%), than before patient contact $(9.7 \%, 95 \%$ CI 5.5-16.6\%) and before clean/aseptic/invasive procedures (7.8\%, 95\% CI 3.4-17.0\%).

\subsection{Environmental Swabs and Hand Swabs}

Overall, 200 environmental swabs and 20 hand swabs were collected and analyzed. From the environmental specimens, 31 (15.5\%) tested positive for at least one of the investigated ARM. CPE were detected in 22 (11.0\%, 95\% CI 7.4-16.1) specimens, ESBL-E in 14 (7.0\%, 95\% CI 4.2-11.4), and methicillin-resistant staphylococci (MRS) in 7 (3.5\%, 95\% CI 1.7-7.0) specimens. MR Staphylococcus aureus were isolated from two hand swabs (10.0\%, 95\% CI 1.77-30.1). Vancomycin-resistant enterococci (VRE) were not detected in any specimens.

The environmental isolates originated from 22 different surfaces from all areas across the clinic, including those where no animals were permitted, such as the staff kitchen, the toilet, and the laboratory (Figure 1). The percentage of ARM positive samples from the environment per day ranged from $10.0 \%$ (95\% CI 4.3-21.4, day 3) to $18.0 \%$ (95\% CI 9.8-30.8, days 1, 2 and 4). The isolates comprised several species and sequence types. The ESBL-E and CPE isolates included Escherichia coli ST961, ST12, ST641, and ST1406; Klebsiella pneumoniae ST219, ST3063, ST857, ST5580, and ST5873; Enterobacter hormaechei; Citrobacter braakii; and Serratia marcescens (Figure 1, Table S1). All CPE carried bla $a_{\mathrm{OXA}-48}$; the ESBL-E carried $b l a_{\mathrm{CTX}-\mathrm{M}-15}$ and/ or $b l a_{\mathrm{SHV}-12}$; the MRS isolates all harbored the mecA gene (Table S1).

To investigate a potential clonal dissemination of isolates and horizontal spread of bla $a_{\mathrm{OXA}-48}$-encoding plasmids within the clinical environment, 12 bla $a_{\mathrm{OXA}-48}$ harboring isolates (1 C. braakii, 4 Enterobacter spp., 2 E. coli (ST961 and ST1406), 4 K. pneumoniae, (2 ST219, ST3063, and ST5873) and 1 S. marcescens) were subjected to whole genome sequencing (WGS) (Table S2). The four Enterobacter spp. isolates were identified as E. hormaechei according rMLST analyses and assigned to ST113 and ST114 (3x) using the Enterobacter cloacae complex MLST scheme. E. cloacae complex clades harboring ST113 and ST114 isolates were previously designated as E. hormaechei subsp. steigerwaltii, and E. hormaechei subsp. xiangfangensis (or E. xiangfangensis), respectively [17,18]. Hybrid assemblies of three isolates (2 K. pneumoniae (ST219 and ST5873), and 1 S. marcescens) which additionally underwent long-read sequencing revealed the presence of identical bla $a_{\mathrm{OXA}-48}$-carrying 63,589 bp IncL plasmids in each isolate. Read-mapping-based approaches confirmed the presence of this pOXA-48-like plasmid in all other sequenced CPE isolates. The plasmid was conserved across the different clones and species, deviating only in E. coli MV-r4-SK2-C by one SNP (T37049A). In the NCBI nucleotide collection, the identical plasmid (63,589 bp; $100 \%$ sequence identity and query cover; ignoring inversions) was found in 44 genomes from global isolates (33 K. pneumoniae, 8 E. coli, and 3 Citrobacter freundii) deriving, amongst others, from humans and the environment of another Swiss veterinary clinic [19]. The plasmid obtained in the veterinary clinic differed structurally by a $5.6 \mathrm{~kb}$ inversion (Figure 2). The genetic structure of the plasmid was described before (pEc_MW04_OXA, [20]) and did not contain antimicrobial resistance genes other than bla $a_{\mathrm{OXA}-48}$.

WGS also demonstrated a clonal spread of CPE across different sources and sampling days in the clinic environment: three of the four sequenced E. hormaechei belonged to ST114 and differed by $\leq 7$ pairwise whole genome SNPs. The three isolates were found on three distinct surfaces in different areas of the clinic and collected over a period of 12 days. Similarly, the two sequenced K. pneumoniae ST219 isolates differed by four whole genome SNPs, suggesting a common origin. ST219 was found throughout the clinic and on every sampling day (Figure 1). 


\begin{tabular}{|c|c|c|c|c|}
\hline Alcohol & $\begin{array}{c}\text { Day } 1 \\
\text { a }\end{array}$ & Day 3 & Day 8 & Day 12 \\
\hline Bowl & & & घ & \\
\hline Cat cage & $\begin{array}{l}\text { ST219 } \\
\text { ST114 } \\
\text { ST219 }\end{array}$ & $\Delta$ & & ST961 (ST12Cplx) \\
\hline Centrifuge & & ST1406 & & \\
\hline Door & - & & & \\
\hline Drain & ST219 & : & & $\begin{array}{l}\text { ST219 } \\
\text { ST } 219 \\
\text { ST219 } \\
\text { ST219 }\end{array}$ \\
\hline Floor & - & 8 & & ST5873 \\
\hline Fridge & & 8 & & \\
\hline Hand & & & & 블 \\
\hline Heating device & & 8 & & \\
\hline Keyboard & - & & & 8 \\
\hline Microwave & & & - & \\
\hline 02 device & & & & - \\
\hline Otoscope & - & & $\mathbf{A}$ & \\
\hline Scale (touch display) & $\begin{array}{l}\text { ST5580 } \\
\text { ST114 }\end{array}$ & & & \\
\hline SpO2 device & & sT113 & & \\
\hline $\begin{array}{l}\text { Table with drain and } \\
\text { shower system }\end{array}$ & & $\AA_{\text {ST219 }}$ & & $\overbrace{}^{\text {ST114 }}$ \\
\hline Thermometer & & & & i \\
\hline Toilet (door handle) & & & $A^{\mathrm{ST} 219}$ & \\
\hline Ultrasound device & & & i & \\
\hline Washing machine & : & & & \\
\hline Water tap & & ¿ ST219 & & $:$ \\
\hline
\end{tabular}

Figure 1. Timeline of CPE, ESBL-E, and MRS isolated from the clinical environment. Each column refers to one sampling day. Each horizontal line refers to a specimen obtained from the same environmental surface over time. Isolates of the same surface on the same day derived from subcultures of the same samples. Negative test results are omitted. The sequence types are indicated at the right side of the symbols for the Escherichia coli, Klebsiella pneumoniae, and the Enterobacter hormaechei isolates for which whole genome sequencing was conducted. Circles indicate CPE, triangles indicate ESBL-E, and squares indicate MRS. The color of the symbols indicate: purple, Staphylococcus spp.; orange, Serratia maracescens; yellow, Citrobacter braakii; blue, Enterobacter hormaechei; red, Escherichia coli; and green, Klebsiella pneumoniae. Abbreviations: ST, sequence type; CPE, carbapenemase-producing Enterobacteriaceae; ESBL-E, extended spectrum beta-lactamase-producing Enterobacteriaceae; and MRS, methicillin-resistant staphylococci.

The number of antimicrobial resistance genes identified in the entire genome of the sequenced CPE isolates ranged from only one gene (bla $\left.a_{\mathrm{OXA}-48}\right)$ in each of the two E. coli isolates, to 17 genes in both clones associated with spread, i.e., E. hormaechei ST114 and K. pneumoniae ST219 (Table S2). These included bla $a_{\mathrm{CTX}-\mathrm{M}-15}$ and fosA in the two K. pneumoniae ST117 isolates, and mcr-9 and fosA in the three E. hormaechei ST114 isolates, amongst others.

AmpC-type $\beta$-lactamase genes were identified in the C. brakii aisolate $\left(b l a_{\mathrm{CMY}}-101\right)$, in the E. hormaechei ST113 isolate (bla $\left.a_{\mathrm{ACT}-15}\right)$, in the three E. hormaechei ST114 isolates (bla $\left.a_{\mathrm{ACT}-16}\right)$, and in the $S$. marcescens isolate (bla $\left.a_{\mathrm{SRT}-2}\right)$. BLAST searches of the NCBI nucleotide collection using contigs harboring the different AmpC-type $\beta$-lactamase genes as queries suggested chromosomal locations in all six cases. Likewise, bla $\mathrm{SHV}$ genes identified in the four $K$. 
pneumoniae isolates were located in the chromosome. Hybrid assemblies of K. pneumoniae MV-u1-SK2-O, K. pneumoniae MV-v4-SK2-O, and S. marcescens MV-u1-SK1-O revealed the presence of five, two, and two plasmids, respectively. The (co-)location of antimicrobial resistance genes in the chromosome or plasmids are shown in Table S3.

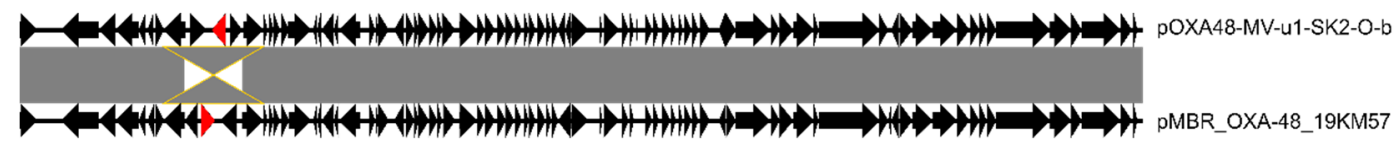

Figure 2. Genetic similarity of $b l a_{\mathrm{OXA}-48}$-harboring plasmids from this and a previous study. Comparison of pOXA48-MV-u1-SK2-O-b (CP085868.1) from Klebsiella pneumoniae ST219 isolate OXA48MV-u1-SK2-O and the publicly available sequence of pMBR_OXA-48_19KM57 (CP039950.1) from Klebsiella pneumoniae ST11 isolate 19KM57, which was found in a different veterinary clinic in Switzerland [19]. Shaded boxes between sequences indicate homologous regions (100\% sequence identity). The $b l a_{\mathrm{OXA}} 48$ gene (colored in red) was part of a $5.6 \mathrm{~kb}$ region (box outlined in yellow) flanked by IS10A insertion elements. This region was inverted in pOXA48-MV-u1-SK2-O-b when compared to pMBR_OXA-48_19KM57. The figure was generated with Easyfig 2.1 [21].

Resistance profiles were determined for all ESBL-E and CPE isolates collected in this study (Table S1). All ESBL-E and CPE isolates showed resistance to ampicillin and cephazolin. Most strains were resistant to cefotaxime, amoxicillin-clavulanic acid, sulfamethoxazole trimethoprim, azithromycin, streptomycin, and tetracyclin. One isolate was resistant to fosfomycin. Of the $\mathrm{CPE}$, two isolates were resistant to ertapenem. One of these strains was additionally resistant to meropenem and imipenem.

\section{Discussion}

Our study documents an extensive environmental contamination with CPE, ESBL-E, and MRS isolates in a companion animal clinic in Switzerland. A total of $15.5 \%$ of the environmental specimens harbored at least one of the investigated ARM. From 11.0\% of the environmental specimens, a CPE was isolated. This is already the second report of a CPE dissemination in a companion animal clinic in Switzerland. Recently, a CPE outbreak caused by $E$. coli harboring the carbapenemase gene bla $a_{\mathrm{OXA}-181}$ was documented in a companion animal clinic in Switzerland. During the outbreak, 21.6\% of the hospitalized dogs and cats were colonized with E. coli bla $a_{\mathrm{OXA}-181}$ after two days of hospitalization, whereas only one of the tested animals was colonized at the time of admission (0.75\%) [12]. Of concern, carriage of a closely related bla $a_{\mathrm{OXA1}}$-positive E. coli was also detected in a staff member of the clinic [22]. Furthermore, CP K. pneumoniae (ST11, bla $a_{\mathrm{OXA}-48}$ ) was found in $22 \%$ of the environmental sampling sites collected in the ICU and the emergency room in this institution [23]. In contrast to the aforementioned outbreak, the successful spread in our study relied not only on clonal, but also on plasmidic dissemination of the IncL plasmid. Based on SNP analyses and MLST, two dissemination clusters were detected in the present study, namely E. hormaechei ST114 and K. pneumoniae ST219. Near-identical strains were isolated from different sampling sites, indicating the presence of a common source. Identical IncL OXA-48 plasmids were found in 11 of the 12 sequenced isolates, which belonged to 5 different bacterial species. The identified IncL plasmid has been isolated world-wide from various sources, including veterinary and human healthcare settings $[5,6,19,20,24]$. Of note, the IncL plasmid was compositionally identical to a plasmid isolated from another Swiss veterinary referral hospital (5.6 kb inversion difference [19]). A connection between these two clinics cannot be ruled out because animal patients are frequently referred between the two institutions. The repeated occurrence of this plasmid in companion animal clinics might contribute to the spread of CPE, posing a threat to public health.

Contamination in companion animal clinics may vary across sampling days, as has been previously reported [25]. This was also evident in the present study, where ARM contamination rates ranged from 10.0-18.0\% over the 4 sampling days. In our study, ESBL-E was found in $7.0 \%$ of the 200 samples collected from the clinical environment. A recent 
study in five Swiss companion animal clinics found ESBL-E contamination in $0-2 \%$ of the environmental specimens [23]. ESBL-E were the most common ARM acquired by dogs and cats during hospitalization in four clinics in Switzerland [26] and were recently shown to spread to the household environment and to the animal owners after patient discharge [25]. In the latter study, transmission chains for four different high-risk human pathogenic strains of ESBL-E were documented over a 45-day observation period in a companion animal ICU. After discharge, the animal owners showed colonization with closely related ESBL-E strains and extensive contamination of the household environment with ESBL-E was documented. Transmission of CPE and ESBL-E between owners and their pets was also reported in other studies $[27,28]$, which underlines the public health concern of ARM dissemination by companion animal veterinary institutions [25,28-34].

ARM-positive samples were commonly retrieved from areas with no patient contact, such as the kitchen, the laboratory, and the toilets. In a recent study conducted at a companion animal shelter, these areas were reported to have a high bacterial contamination [35]. Thus, areas with little to no patient contact in a veterinary clinic could pose a reservoir for ARM and a focus should also be placed on these locations when implementing cleaning and disinfection protocols. The conducted audit revealed a low overall IPC score in the investigated clinic ( $58 \%$ of the maximum IPC score). This goes in line with results from the previous study, where three companion animal clinics in Switzerland with extensive ARM contamination reached only $28-52 \%$ of the maximum IPC score [23]. Of note, hand hygiene infrastructure was absent in many patient areas in the herein described clinic, and hand hygiene compliance was worryingly low, with an overall compliance of only $14.9 \%$. This compliance is even lower than recently reported in several companion animal institutions in Switzerland and companion animal clinics abroad, which reported a hand hygiene compliance of $14-42 \%$ [36-40]. Hand hygiene is regarded a key component to interrupt transmission chains for ARM and other pathogens in hospital settings [41-44]. The very low hand hygiene compliance before clean/aseptic/invasive procedures $(7.8 \%)$ and before patient contact $(9.7 \%)$ in this clinic poses the patients at greater risk to acquire ARM through the hands of the healthcare workers.

The present study also has its limitations. For one, the IPC scoring system is based on a consensus which might be subjective to interpretation. Additionally, animal patients and healthcare workers were not sampled. However, recent studies have already documented closely related ARM, including CPE, in patients, healthcare workers, and the clinical environment of companion animal clinics $[12,22,26,45]$. The number of hand swabs investigated in the present study was limited, and the prevalence of ARM might thus be over- or underestimated.

\section{Materials and Methods}

\subsection{Study Set-Up and IPC Evaluation}

IPC standards in the clinic were evaluated by direct audit as previously described [23] and an adapted IPC audit protocol that included fifteen areas of IPC was applied [46]. The audit assessed general IPC management, staff education, cleaning/disinfection, management of waste, vector control, equipment in examination rooms, isolation measures, handling of patients with ARM, hand hygiene equipment, personal hygiene, protection of employees, protective clothing, medication, use of antimicrobials, and miscellaneous (Table S4). A scoring system (0: not fulfilled; 1: partially fulfilled; 2: completely fulfilled) was applied, and a total score calculated as described [23]. Participation in the study was voluntary and was not reimbursed. After the IPC audit, the sampling, and the hand hygiene observations were completed, the participating clinic received a written report of all results, highlighting the IPC deficits and an action plan for IPC and hand hygiene implementation.

\subsection{Hand Hygiene Evaluation}

Hand hygiene compliance was evaluated according to the WHO five moments of hand hygiene (after body fluid exposure risk, after patient contact, after touching the patient's sur- 
roundings, before clean/aseptic/invasive procedures, and before patient contact) using the CleanHands application version February 2021 (Swissnoso, National Centre for Infection Prevention) as previously described [37]. Briefly, the CleanHands application was used to evaluate hand hygiene (i.e., carried out or not) in four different clinical areas: consultation rooms, wards, ICU, and pre-operation preparation area. Hand hygiene compliance across professional groups (veterinarians, nurses, and others, i.e., personnel not allocated to the aforementioned categories) was analyzed. According to WHO guidelines, glove usage was not categorized as a hand hygiene event. All observations were carried out by the same observer over a period of one week; the observer was previously trained by an experienced observer [36]. After digital recording, the data was extracted from the software as Excel files for further statistical analyses. Non-coded hand hygiene observations, i.e., those that could not be matched to one of the five moments of hand hygiene, were excluded from statistical analysis.

\subsection{Statistical Analysis}

The commercially available GraphPad PRISM ${ }^{\circledR}$ software (San Diego, CA, USA) was used for statistical analysis. Descriptive statistics were conducted for the hand hygiene compliance $(\%$, number of correct hand hygiene events per total number of observed hand hygiene events), and binomial confidence intervals for hand hygiene compliance were calculated using the hybrid Wilson/Brown method [47]. Contingency tables were calculated using the chi-square test. Significance was set at $p<0.05$.

\subsection{Microbiological Evaluation}

Swab samples from a predetermined list of surfaces (Table S5) and from veterinary employees' hands were collected on four different days over a two-week period. As previously described [37], hand swabs of the entire dominant hand palm, fingers, and thumb were collected before and after patient contact using a sterile cotton swab moisturized with $0.85 \%$ saline solution. If gloves were worn, hand swabs were taken from the gloved hand directly, before and after patient contact. To reduce potential observer bias, hand swabs were taken during busy daily procedures and in areas with a high density of patients and personnel. The healthcare workers were approached immediately before animal contact without any prior announcement. A coded sample collection procedure was used, and hence, no personal data was collected from the study participants to ensure that employees did not feel obliged to change their hand hygiene behavior. All study participants gave written informed consent, and the study protocol was approved by the Swiss Ethics Committees on research involving humans (approval no. 2019-00768).

Hand swabs were processed immediately after sample collection, as previously published [37]. The swabs were homogenized for $60 \mathrm{~s}$ in $10 \mathrm{~mL}$ peptone water (BioRad, Hercules, CA, USA) using in a Stomacher ${ }^{\circledR} 400$ (Seward, Worthing, UK). The homogenate of each sample was thereafter enriched $\left(37^{\circ} \mathrm{C}, 24 \mathrm{~h}\right)$, followed by selective enrichment for ESBL-E and CPE in Enterobacteriaceae enrichment broth (Oxoid, Hampshire, UK), in BHI (BioRad, Hercules, CA, USA) with 6.5\% saline solution for VRE, and additionally in Mueller Hinton broth (Oxoid, Hampshire, UK) with 6.5\% saline solution, followed by an enrichment in tryptone soy broth (Becton Dickinson, Allschwil, Switzerland) with $4 \mathrm{mg} / \mathrm{L}$ cefoxitin and $75 \mathrm{mg} / \mathrm{L}$ aztreonam for the detection of MRS. ESBL-E were screened by using the chromogenic medium Brilliance ${ }^{\mathrm{TM}}$ ESBL Agar (Oxoid, Hampshire, UK), CPE by using chromID ${ }^{\circledR}$ CARBA SMART Bi-Plate-Agar (bioMérieux, Marcy-l'Étoile, France), VRE by using the Brilliance ${ }^{\mathrm{TM}}$ VRE Agar (Oxoid, Hampshire, UK)m and MRS by using the Brilliance ${ }^{\mathrm{TM}}$ MRSA2 Agar (Oxoid, Hampshire, UK), according to the manufacturer's instructions. Species identification was conducted by using matrix-assisted laser desorption/ionization time-of-flight mass spectrometry (MALDI-TOF-MS, Bruker Daltronics, Bremen, Germany).

Polymerase chain reaction (PCR) was carried out to screen for the presence of genes encoding bla $a_{\mathrm{CTX}-\mathrm{M}}$ group enzymes, bla $a_{\mathrm{SHV}}$, and bla $a_{\mathrm{TEM}}$, as previously described [48-51]. 
PCR targeting $b l a_{\mathrm{VIM}}, b l a_{\mathrm{KPC}}, b l a_{\mathrm{OXA}-48}$-like, and $b l a_{\mathrm{NDM}}$ genes was carried out using custom synthesized primers (Microsynth, Balgach, Switzerland) and conditions published previously [52,53]. Multiplex PCR for the presence of $\operatorname{van} A$, vanB, and $\operatorname{van} C_{1,2,3}$ was conducted as previously described using custom synthesized primers (Microsynth, Balgach, Switzerland) [54]. PCR for the presence of mecA and mecC was conducted using custom synthesized primers (Microsynth, Balgach, Switzerland), as previously described [55,56].

Antimicrobial susceptibility testing was carried out for all ESBL-E and CPE isolates as previously described [25]. Antimicrobial susceptibility testing was performed for Enterobacteriaceae in accordance with the Clinical and Laboratory Standards Institute (CLSI) performance standards [57] using the disk-diffusion method on Mueller Hinton plates (Oxoid, Hampshire, UK) and the 16 antibiotics: ampicillin (AM), amoxicillin with clavulanic acid (AMC), azithromycin (AZM), cefazolin (CZ), cefepime (FEP), cefotaxime (CTX), chloramphenicol (C), ciprofloxacin (CIP), fosfomycin (FOS), gentamicin (G), kanamycin (K), nalidixic acid (NA), nitrofurantoin (F/M), streptomycin (S), sulfamethoxazole trimethoprim (SXT), and tetracycline (TE) (Becton Dickinson, Allschwil, Switzerland). Results were interpreted according to CLSI standards [57]. For azithromycin, an inhibition zone of $\leq 12 \mathrm{~mm}$ was interpreted as resistant. In addition, the minimal inhibitory concentrations of the carbapenem antibiotics ertapenem, imipenem, and meropenem were determined for all CPE isolates.

For MRS isolates, antimicrobial susceptibility profiling was performed using the automated VITEK ${ }^{\circledR}$ two compact system (bioMérieux, Marcy l'Etoile, France) with the AST-GP80 susceptibility testing card (bioMérieux, Nürtingen, Germany).

\subsection{Whole-Genome Sequencing and Genomic Analyses}

A subset of 12 CPE isolates underwent Illumina short-read sequencing, of which 3 were additionally subjected to nanopore long-read sequencing (Table S2). For short-read sequencing, genomic DNA was extracted using the DNeasy Blood \& Tissue Kit (Qiagen, Hilden, Germany). Libraries were prepared using the Nextera DNA Flex Library Preparation Kit (Illumina) and sequenced on the Illumina MiniSeq platform with $2 \times 150 \mathrm{bp}$ paired-end chemistries. Draft genomes were assembled using SPAdes v3.14.1 [58] implemented in shovill v1.1.0 (github.com/tseemann/shovill; accessed on 1 October 2021) [59]. For nanopore sequencing, genomic DNA was extracted with the MasterPure Complete DNA and RNA Purification Kit (Lucigen). Multiplex libraries were prepared using the SQK-LSK109 ligation sequencing kit with the EXP-NBD114 native barcoding expansion kit (Oxford Nanopore Technologies). Libraries were sequenced on a MinION Mk1B device using the FLO-MIN106 (R9) flow cell (Oxford Nanopore Technologies). Hybrid assemblies were produced with Unicycler 0.4.8 [60,61]. Taxonomy was assigned using rMLST [61]. Antimicrobial resistance genes and plasmid replicons were identified using abricate 1.0.1 (github.com/tseemann/abricate; accessed on 1 October 2021), in conjunction with the ResFinder [62] and PlasmidFinder [63] database, respectively. Multi-locus sequence types (MLST) were determined using mlst 2.19 .0 (github.com/tseemann/mlst; accessed on 1 October 2021). Whole genome SNPs among K. pneumoniae ST219 and E. hormaechei ST114 isolates were detected from Illumina read data using the CFSAN SNP pipeline v2.2.1 [64], with assemblies of isolates MV-u1-SK2-O and MV-oo4-C as references, respectively.

The variability of pOXA-48-like plasmids in isolates only subjected to short-read sequencing was determined by SNP detection using the CFSAN pipeline with pOXA48-MVu1-SK2-O-b (circular plasmid from the hybrid assembly of isolate MV-u1-SK2-O, accession CP085868) as reference. Plasmid coverage and identified SNPs were confirmed by readmapping and variant detection implemented in CLC Genomics Workbench 21.0.4.

\subsection{Data Availability}

Sequencing data and genome assemblies generated as part of this study are available under BioProject no. PRJNA774102. Genome accession numbers of all investigated isolates are listed in Table S2. 


\section{Conclusions}

Our results indicate that plasmidic dissemination of $b l a_{\text {OXA-48 }}$ in companion animal clinics may occur and poses a worrisome threat to public health. The IncL plasmid was found in five different bacterial species and isolated on each of four sampling days in a companion animal clinic in Switzerland. IPC standards, hand hygiene equipment, and hand hygiene adherence were largely insufficient in the clinic, and improvement in these aspects might support the containment of the ARM dissemination. Areas without patient contact were also commonly contaminated, and cleaning and disinfection protocols should not omit these areas, as they might represent a reservoir for ARM. The study highlights the need to develop and implement evidence-based IPC concepts and hand hygiene trainings in veterinary clinics to prevent the spread of ARM into the community. The ongoing project will analyze the effect of IPC implementation as part of an intervention strategy.

Supplementary Materials: The following supporting information can be downloaded at: https: //www.mdpi.com/article/10.3390/antibiotics11020213/s1, Table S1: Antimicrobial resistant microorganisms isolated from the clinical environment; Table S2: Sequencing data for strains isolated in this study; Table S3: Co-location of antimicrobial resistance genes identified in hybrid assemblies of three selected carbapenem-producing isolates; Table S4: Criteria applied for the audit scoring; Table S5: List of environmental sampling sites in the small animal clinic.

Author Contributions: R.S., B.W. and K.S. contributed to the design of the study. K.S. conducted the sampling and data collection. B.W. and K.S. performed the IPC audit. K.S. isolated and identified the strains and performed the microbiological work. M.B. carried out the genomic analysis and sequencing. R.S., M.B. and K.S. interpreted the bacteriological and molecular the data, B.W. and K.S., the IPC and hand hygiene data. K.S. wrote the manuscript, and R.S., B.W. and M.B. edited the manuscript. This study was part of the Ph.D. project of Kira Schmitt. All authors have read and agreed to the published version of the manuscript.

Funding: This study was financed by the Swiss Federal Food Safety and Veterinary Office (FSVO Grant no. 1.21.q "Effect of the implementation of infection prevention and control concepts and hand hygiene campaigns in companion animal clinics in Switzerland") and the Swiss Association for Small Animal Medicine.

Institutional Review Board Statement: The study was conducted in accordance with the Declaration of Helsinki, and approved by the Swiss Ethics Committees on research involving humans (approval no. 2019-00768).

Informed Consent Statement: Informed consent was obtained from all subjects involved in the study.

Data Availability Statement: Data is available upon reasonable request.

Acknowledgments: The authors are grateful to the companion animal clinic for the participation in the present study. Additionally, we thank Nicole Cernela for her help in sequencing.

Conflicts of Interest: The authors declare no conflict of interest.

\section{References}

1. United Nations. Resolution A/RES/71/3: Political Declaration of the High-Level Meeting of the General Assembly on Antimicrobial Resistance. In Proceedings of the Seventy-First Session of the United Nations General Assembly, New York, NY, USA, 5 October 2016.

2. Interagency Coordination Group on Antimicrobial Resistance. No Time to Wait: Securing the Future from Drug-Resistant Infections Report to the Secretary-General of the United Nation; United Nations: New York, NY, USA, 2019.

3. World Health Organization. WHO List of Critically Important Antimicrobials for Human Medicine (WHO CIA List); World Health Organization: Geneva, Switzerland, 2017.

4. Potron, A.; Poirel, L.; Nordmann, P. Derepressed transfer properties leading to the efficient spread of the plasmid encoding carbapenemase OXA-48. Antimicrob. Agents Chemother. 2014, 58, 467-471. [CrossRef]

5. Ledda, A.; Cummins, M.; Shaw, L.; Jauneikaite, E.; Cole, K.; Lasalle, F.; Barry, D.; Rosmarin, C.; Anaraki, S.; Wareham, D.; et al. Hospital outbreak of carbapenem-resistant Enterobacteriales associated with an OXA-48 plasmid carried mostly by Escherichia coli ST399. bioRxiv 2020. [CrossRef] 
6. Shaidullina, E.; Shelenkov, A.; Yanushevich, Y.; Mikhaylova, Y.; Shagin, D.; Alexandrova, I.; Ershova, O.; Akimkin, V.; Kozlov, R.; Edelstein, M. Antimicrobial resistance and genomic characterization of OXA-48-and CTX-M-15-co-producing hypervirulent Klebsiella pneumoniae ST23 recovered from nosocomial outbreak. Antibiotics 2020, 9, 862. [CrossRef]

7. Albiger, B.; Glasner, C.; Struelens, M.J.; Grundmann, H.; Monnet, D.L.; The European Survey of Carbapenemase-Producing Enterobacteriaceae (EuSCAPE) Working Group. Carbapenemase-producing Enterobacteriaceae in Europe: Assessment by national experts from 38 countries, May 2015. Eurosurveillance 2015, 20, 30062. [CrossRef]

8. Hidron, A.I.; Edwards, J.R.; Patel, J.; Horan, T.C.; Sievert, D.M.; Pollock, D.A.; Fridkin, S.K. National Healthcare Safety Network Team and Participating National Healthcare Antimicrobial-resistant pathogens associated with healthcare-associated infections: Annual summary of data reported to the National Healthcare Safety Network at the Centers for Disease Control and Prevention, 2006-2007. Infect. Control Hosp. Epidemiol. 2008, 29, 996-1011.

9. Weiner, L.M.; Webb, A.K.; Limbago, B.; Dudeck, M.A.; Patel, J.; Kallen, A.J.; Edwards, J.R.; Sievert, D.M. Antimicrobial-resistant pathogens associated with healthcare-associated infections: Summary of data reported to the National Healthcare Safety Network at the Centers for Disease Control and Prevention, 2011-2014. Infect. Control Hosp. Epidemiol. 2016, 37, 1288-1301. [CrossRef]

10. Marí-Almirall, M.; Cosgaya, C.; Pitart, C.; Viñes, J.; Muñoz, L.; Campo, I.; Cuscó, A.; Rodríguez-Serna, L.; Santana, G.; Del Río, A.; et al. Dissemination of NDM-producing Klebsiella pneumoniae and Escherichia coli high-risk clones in Catalan healthcare institutions. J. Antimicrob. Chemother. 2020, 76, 1-10. [CrossRef]

11. Poirel, L.; Lienhard, R.; Potron, A.; Malinverni, R.; Siegrist, H.H.; Nordmann, P. Plasmid-mediated carbapenem-hydrolysinglactamase KPC-2 in a Klebsiella pneumoniae isolate from Switzerland. J. Antimicrob. Chemother. 2011, 66, 675-676. [CrossRef]

12. Nigg, A.; Brilhante, M.; Dazio, V.; Clément, M.; Collaud, A.; Brawand, S.G.; Willi, B.; Endimiani, A.; Schuller, S.; Perreten, V. Shedding of OXA-181 carbapenemase-producing Escherichia coli from companion animals after hospitalisation in Switzerland: An outbreak in 2018. Eurosurveillance 2019, 24, 1-12. [CrossRef]

13. Gentilini, F.; Turba, M.E.; Pasquali, F.; Mion, D.; Romagnoli, N.; Zambon, E.; Terni, D.; Peirano, G.; Pitout, J.D.D.; Parisi, A.; et al. Hospitalized pets as a source of carbapenem-resistance. Front. Microbiol. 2018, 9, 2872. [CrossRef]

14. Cocca, G.; Piva, S.; Magno, S.D.; Scarpellini, R.; Giacometti, F.; Serraino, A.; Giunti, M. Prevalence and Patterns of Antimicrobial Resistance among Escherichia coli and Staphylococcus spp. in a Veterinary University Hospital. Vet. Sci. 2021, 8, 308. [CrossRef] [PubMed]

15. Pulss, S.; Stolle, I.; Stamm, I.; Leidner, U.; Heydel, C.; Semmler, T.; Prenger-Berninghoff, E.; Ewers, C. Multispecies and clonal dissemination of OXA-48 carbapenemase in Enterobacteriaceae from companion animals in Germany, 2009-2016. Front. Microbiol. 2018, 9, 1265. [CrossRef]

16. Smith, A.; Wayne, A.S.; Fellman, C.L.; Rosenbaum, M.H. Usage patterns of carbapenem antimicrobials in dogs and cats at a veterinary tertiary care hospital. J. Vet. Intern. Med. 2019, 33, 1677-1685. [CrossRef]

17. Brilhante, M.; Gobeli Brawand, S.; Endimiani, A.; Rohrbach, H.; Kittl, S.; Willi, B.; Schuller, S.; Perreten, V. Two high-risk clones of carbapenemase-producing Klebsiella pneumoniae that cause infections in pets and are present in the environment of a veterinary referral hospital. J. Antimicrob. Chemother. 2021, 76, 1140-1149. [CrossRef] [PubMed]

18. Kumwenda, G.P.; Sugawara, Y.; Akeda, Y.; Matsumoto, Y.; Motooka, D.; Tomono, K.; Hamada, S. Genomic features of plasmids coding for KPC-2, NDM-5 or OXA-48 carbapenemases in Enterobacteriaceae from Malawi. J. Antimicrob. Chemother. 2021, 76, 267-270. [CrossRef]

19. Pot, M.; Guyomard-Rabenirina, S.; Couvin, D.; Ducat, C.; Enouf, V.; Ferdinand, S.; Gruel, G.; Malpote, E.; Talarmin, A.; Breurec, S.; et al. Dissemination of extended-spectrum- $\beta$-lactamase-producing enterobacter cloacae complex from a hospital to the nearby environment in guadeloupe (French West Indies): ST114 lineage coding for a successful inchi2/st1 plasmid. Antimicrob. Agents Chemother. 2021, 65, e02146-20. [CrossRef]

20. Sullivan, M.J.; Petty, N.K.; Beatson, S.A. Easyfig: A genome comparison visualizer. Bioinformatics 2011, 27, 1009-1010. [CrossRef]

21. Chavda, K.D.; Chen, L.; Fouts, D.E.; Sutton, G.; Brinkac, L.; Jenkins, S.G.; Bonomo, R.A.; Adams, M.D.; Kreiswirth, B.N Comprehensive genome analysis of carbapenemase-producing Enterobacter spp.: New insights into phylogeny, population structure, and resistance mechanisms. mBio 2016, 7, e02093-16. [CrossRef]

22. Endimiani, A.; Brilhante, M.; Bernasconi, O.J.; Perreten, V.; Schmidt, J.S.; Dazio, V.; Nigg, A.; Gobeli Brawand, S.; Kuster, S.P.; Schuller, S.; et al. Employees of Swiss veterinary clinics colonized with epidemic clones of carbapenemase-producing Escherichia coli. J. Antimicrob. Chemother. 2020, 75, 766-768. [CrossRef]

23. Schmidt, J.S.; Kuster, S.P.; Nigg, A.; Dazio, V.; Brilhante, M.; Rohrbach, H.; Bernasconi, O.J.; Büdel, T.; Campos-Madueno, E.I.; Gobeli Brawand, S.; et al. Poor infection prevention and control standards are associated with environmental contamination with carbapenemase-producing Enterobacterales and other multidrug-resistant bacteria in Swiss companion animal clinics. Antimicrob. Resist. Infect. Control. 2020, 9, 93. [CrossRef]

24. Hendrickx, A.P.A.; Landman, F.; De Haan, A.; Witteveen, S.; Van Santen-Verheuvel, M.G.; Schouls, L.M. bla OXA-48-like genome architecture among carbapenemase-producing Escherichia coli and Klebsiella pneumoniae in the Netherlands DATA SUMMARY. Microb. Genom. 2021, 7, 512.

25. Schmitt, K.; Kuster, S.P.; Zurfluh, K.; Jud, R.S.; Sykes, J.E.; Stephan, R.; Willi, B. Transmission Chains of Extended-Spectrum Beta-Lactamase-Producing Enterobacteriaceae at the Companion Animal Veterinary Clinic-Household Interface. Antibiotics 2021, 10, 171. [CrossRef] 
26. Dazio, V.; Nigg, A.; Schmidt, J.S.; Brilhante, M.; Mauri, N.; Kuster, S.P.; Brawand, S.G.; Schüpbach-Regula, G.; Willi, B.; Endimiani, A.; et al. Acquisition and carriage of multidrug-resistant organisms in dogs and cats presented to small animal practices and clinics in Switzerland. J. Vet. Intern. Med. 2021, 35, 970-979. [CrossRef] [PubMed]

27. Toombs-Ruane, L.J.; Benschop, J.; French, N.P.; Biggs, P.J.; Midwinter, A.C.; Marshall, J.C.; Chan, M.; Drinković, D.; Fayaz, A.; Baker, M.G.; et al. Carriage of Extended-Spectrum-Beta-Lactamase- and AmpC Beta-Lactamase-Producing Escherichia coli Strains from Humans and Pets in the Same Households. Appl. Environ. Microbiol. 2020, 86, 1-15. [CrossRef]

28. Grönthal, T.; Österblad, M.; Eklund, M.; Jalava, J.; Nykäsenoja, S.; Pekkanen, K.; Rantala, M. Sharing more than friendshipTransmission of NDM-5 ST167 and CTX-M-9 ST69 Escherichia coli between dogs and humans in a family, finland, 2015. Eurosurveillance 2018, 23, 1700497. [CrossRef]

29. Zogg, A.L.; Zurfluh, K.; Schmitt, S.; Nüesch-Inderbinen, M.; Stephan, R. Antimicrobial resistance, multilocus sequence types and virulence profiles of ESBL producing and non-ESBL producing uropathogenic Escherichia coli isolated from cats and dogs in Switzerland. Vet. Microbiol. 2018, 216, 79-84. [CrossRef] [PubMed]

30. Nüesch-Inderbinen, M.T.; Baschera, M.; Zurfluh, K.; Hächler, H.; Nüesch, H.; Stephan, R. Clonal diversity, virulence potential and antimicrobial resistance of Escherichia coli causing community acquired urinary tract infection in Switzerland. Front. Microbiol. 2017, 8, 2334. [CrossRef]

31. van den Bunt, G.; Fluit, A.C.; Spaninks, M.P.; Timmerman, A.J.; Geurts, Y.; Kant, A.; Scharringa, J.; Mevius, D.; Wagenaar, J.A.; Bonten, M.J.M.; et al. Faecal carriage, risk factors, acquisition and persistence of ESBL-producing Enterobacteriaceae in dogs and cats and co-carriage with humans belonging to the same household. J. Antimicrob. Chemother. 2020, 75, 342-350. [CrossRef]

32. Silva, M.M.; Sellera, F.P.; Fernandes, M.R.; Moura, Q.; Garino, F.; Azevedo, S.S.; Lincopan, N. Genomic features of a highly virulent, ceftiofur-resistant, CTX-M-8-producing Escherichia coli ST224 causing fatal infection in a domestic cat. J. Glob. Antimicrob. Resist. 2018, 15, 252-253. [CrossRef]

33. Sellera, F.P.; Da Silva, L.C.B.A.; Lincopan, N. Rapid spread of critical priority carbapenemase-producing pathogens in companion animals: A One Health challenge for a post-pandemic world. J. Antimicrob. Chemother. 2021, 76, 2225-2229. [CrossRef]

34. Ewers, C.; Klotz, P.; Leidner, U.; Stamm, I.; Prenger-Berninghoff, E.; Göttig, S.; Semmler, T.; Scheufen, S. OXA-23 and ISAba1-OXA-66 class D $\beta$-lactamases in Acinetobacter baumannii isolates from companion animals. Int. J. Antimicrob. Agents 2017, 49, 37-44. [CrossRef]

35. Horsman, S.; Rynhoud, H.; Zhou, X.; Soares Magalhães, R.J.; Gibson, J.S.; Meler, E. Environmental Recovery of Nosocomial Bacteria in a Companion Animal Shelter Before and After Infection Control Procedures. Front. Vet. Sci. 2021, 7, 1211. [CrossRef]

36. Schmidt, J.S.; Hartnack, S.; Schuller, S.; Kuster, S.P.; Willi, B. Hand hygiene compliance in companion animal clinics and practices in Switzerland: An observational study. Vet. Rec. 2021, 189, e307. [CrossRef] [PubMed]

37. Schmitt, K.; Zimmermann, A.B.E.; Stephan, R.; Willi, B. Hand Hygiene Evaluation Using Two Different Evaluation Tools and Hand Contamination of Veterinary Healthcare Workers in a Swiss Companion Animal Clinic. Vet. Sci. 2021, 8, 260. [CrossRef]

38. Anderson, M.E.; Sargeant, J.M.; Weese, J.S. Video observation of hand hygiene practices during routine companion animal appointments and the effect of a poster intervention on hand hygiene compliance. BMC Vet. Res. 2014, 10, 106. [CrossRef] [PubMed]

39. Shea, A.; Shaw, S. Evaluation of an educational campaign to increase hand hygiene at a small animal veterinary teaching hospital. J. Am. Vet. Med. Assoc. 2012, 240, 61-64. [CrossRef] [PubMed]

40. Smith, J.R.; Packman, Z.R.; Hofmeister, E.H. Multimodal evaluation of the effectiveness of a hand hygiene educational campaign at a small animal veterinary teaching hospital. J. Am. Vet. Med. Assoc. 2013, 243, 1042-1048. [CrossRef]

41. Pittet, D.; Allegranzi, B.; Boyce, J. The World Health Organization Guidelines on Hand Hygiene in Health Care and Their Consensus Recommendations. Infect. Control Hosp. Epidemiol. 2009, 30, 611-622. [CrossRef]

42. Boyce, J.M. MRSA patients: Proven methods to treat colonization and infection. J. Hosp. Infect. 2001, 48, S9-S14. [CrossRef]

43. Marimuthu, K.; Pittet, D.; Harbarth, S. The effect of improved hand hygiene on nosocomial MRSA control. Antimicrob. Resist. Infect. Control 2014, 3, 34. [CrossRef]

44. Sax, H.; Allegranzi, B.; Chraïti, M.-N.; Boyce, J.; Larson, E.; Pittet, D. The World Health Organization hand hygiene observation method. Am. J. Infect. Control. 2009, 37, 827-834. [CrossRef] [PubMed]

45. Wang, M.; Fang, C.; Liu, K.; Wang, L.; Sun, R.; Zhang, R.; Fang, L.; Sun, J.; Liu, Y.; Liao, X. Transmission and molecular characteristics of blaNDM-producing Escherichia coli between companion animals and their healthcare providers in Guangzhou, China. J. Antimicrob. Chemother. 2022, 77, 351-355. [CrossRef] [PubMed]

46. Stull, J.W.; Bjorvik, E.; Bub, J.; Dvorak, G.; Petersen, C.; Troyer, H.L. 2018 AAHA Infection Control, Prevention, and Biosecurity Guidelines. J. Am. Anim. Hosp. Assoc. 2018, 54, 297-326. [CrossRef] [PubMed]

47. Brown, L.D.; Cai, T.T.; DasGupta, A. Interval Estimation for a Binomial Proportion. Stat. Sci. 2001, 16, 101-133. [CrossRef]

48. Zogg, A.L.; Simmen, S.; Zurfluh, K.; Stephan, R.; Schmitt, S.N.; Nüesch-Inderbinen, M. High prevalence of extended-spectrum $\beta$-lactamase producing Enterobacteriaceae among clinical isolates from cats and dogs admitted to a veterinary hospital in Switzerland. Front. Vet. Sci. 2018, 5, 62. [CrossRef] [PubMed]

49. Geser, N.; Stephan, R.; Korczak, B.M.; Beutin, L.; Hächler, H. Molecular identification of extended-spectrum- $\beta$-lactamase genes from Enterobacteriaceae isolated from healthy human carriers in Switzerland. Antimicrob. Agents Chemother. 2012, 56, 1609-1612. [CrossRef]

50. Woodford, N.; Fagan, E.J.; Ellington, M.J. Multiplex PCR for rapid detection of genes encoding CTX-M extended-spectrum $\beta$-lactamases. J. Antimicrob. Chemother. 2006, 57, 154-155. [CrossRef] [PubMed] 
51. Zurfluh, K.; Nüesch-Inderbinen, M.; Morach, M.; Zihler Berner, A.; Hächler, H.; Stephan, R. Extended-spectrum- $\beta$-lactamaseproducing Enterobacteriaceae isolated from vegetables imported from the Dominican Republic, India, Thailand, and Vietnam. Appl. Environ. Microbiol. 2015, 81, 3115-3120. [CrossRef]

52. Poirel, L.; Walsh, T.R.; Cuvillier, V.; Nordmann, P. Multiplex PCR for detection of acquired carbapenemase genes. Diagn. Microbiol. Infect. Dis. 2011, 70, 119-123. [CrossRef]

53. Ellington, M.J.; Kistler, J.; Livermore, D.M.; Woodford, N. Multiplex PCR for rapid detection of genes encoding acquired metallo- $\beta$-lactamases. J. Antimicrob. Chemother. 2007, 59, 321-322. [CrossRef]

54. Dutka-Malen, S.; Evers, S.; Courvalin, P. Detection of glycopeptide resistance genotypes and identification to the species level of clinically relevant enterococci by PCR. J. Clin. Microbiol. 1995, 33, 24-27. [CrossRef]

55. Mehrotra, M.; Wang, G.; Johnson, W.M. Multiplex PCR for detection of genes for Staphylococcus aureus enterotoxins, exfoliative toxins, toxic shock syndrome toxin 1, and methicillin resistance. J. Clin. Microbiol. 2000, 38, 1032-1035. [CrossRef] [PubMed]

56. Stegger, M.; Andersen, P.S.; Kearns, A.; Pichon, B.; Holmes, M.A.; Edwards, G.; Laurent, F.; Teale, C.; Skov, R.; Larsen, A.R. Rapid detection, differentiation and typing of methicillin-resistant Staphylococcus aureus harbouring either mecA or the new mecA homologue mecALGA251. Clin. Microbiol. Infect. 2012, 18, 395-400. [CrossRef]

57. CLSI. Performance Standards for Antimicrobial Susceptibility Testing, 28th ed.; CLSI: Wayne, PA, USA, 2018.

58. Bankevich, A.; Nurk, S.; Antipov, D.; Gurevich, A.A.; Dvorkin, M.; Kulikov, A.S.; Lesin, V.M.; Nikolenko, S.I.; Pham, S.; Prjibelski, A.D.; et al. SPAdes: A new genome assembly algorithm and its applications to single-cell sequencing. J. Comput. Biol. 2012, 19, 455-477. [CrossRef] [PubMed]

59. Seemann, T. Shovill. Available online: https://github.com/tseemann/shovill (accessed on 1 October 2021).

60. Wick, R.R.; Judd, L.M.; Gorrie, C.L.; Holt, K.E. Unicycler: Resolving bacterial genome assemblies from short and long sequencing reads. PLoS Comput. Biol. 2017, 13, e1005595. [CrossRef] [PubMed]

61. Jolley, K.A.; Bliss, C.M.; Bennett, J.S.; Bratcher, H.B.; Brehony, C.; Colles, F.M.; Wimalarathna, H.; Harrison, O.B.; Sheppard, S.K.; Cody, A.J.; et al. Ribosomal multilocus sequence typing: Universal characterization of bacteria from domain to strain. Microbiology 2012, 158, 1005-1015. [CrossRef]

62. Zankari, E.; Hasman, H.; Cosentino, S.; Vestergaard, M.; Rasmussen, S.; Lund, O.; Aarestrup, F.M.; Larsen, M.V. Identification of acquired antimicrobial resistance genes. J. Antimicrob. Chemother. 2012, 67, 2640-2644. [CrossRef]

63. Carattoli, A.; Zankari, E.; Garciá-Fernández, A.; Larsen, M.V.; Lund, O.; Villa, L.; Aarestrup, F.M.; Hasman, H. In Silico detection and typing of plasmids using plasmidfinder and plasmid multilocus sequence typing. Antimicrob. Agents Chemother. 2014, 58, 3895-3903. [CrossRef]

64. Davis, S.; Pettengill, J.B.; Luo, Y.; Payne, J.; Shpuntoff, A.; Rand, H.; Strain, E. CFSAN SNP pipeline: An automated method for constructing snp matrices fromnext-generation sequence data. PeerJ Comput. Sci. 2015, 1, e20. [CrossRef] 